\title{
Different Effects of Voluntary and Involuntary Attention on EEG Activity in the Gamma Band
}

\author{
Ayelet N. Landau, ${ }^{1}$ Michael Esterman, ${ }^{1,2}$ Lynn C. Robertson, ${ }^{1,2}$ Shlomo Bentin, ${ }^{3}$ and William Prinzmetal ${ }^{1}$ \\ ${ }^{1}$ University of California, Berkeley, California 94720, ${ }^{2}$ Department of Veterans Affairs, Martinez, California 94553, and ${ }^{3}$ Hebrew University, \\ 91905 Jerusalem, Israel
}

\begin{abstract}
Previous studies have shown that EEG activity in the gamma range can be modulated by attention. Here, we compared this activity for voluntary and involuntary spatial attention in a spatial-cueing paradigm with faces as targets. The stimuli and trial timing were kept constant across attention conditions with only the predictive value of the cue changing. Gamma-band response was linked to voluntary shifts of attention, but not to the involuntary capture of attention. The presence of increased gamma responses for the voluntary allocation of attention, and its absence in cases of involuntary capture suggests that the neural mechanisms governing these two types of attention are different. Moreover, these data allow a description of the temporal dynamics contributing to the dissociation between voluntary and involuntary attention. The distribution of this correlate of voluntary attention is consistent with a top-down process involving contralateral anterior and posterior regions.
\end{abstract}

Key words: faces; attention; cueing; EEG; electroencephalogram; gamma; visual selectivity

\section{Introduction}

Behavioral evidence suggests that spatial attention can be summoned in at least two ways. One is goal directed and engages top down control mechanisms, whereas the other is automatic and independent of the task. The former is often referred to as endogenous or voluntary attention (Posner, 1978) and the latter as exogenous or involuntary attention (Jonides, 1981).

Ample evidence, using the spatial-cueing paradigm (Posner, 1978) (see Fig. 1a), indicates that for both voluntary and involuntary attention, targets are detected and discriminated faster at validly cued locations compared with invalidly cued locations (the "validity effect"). It is often assumed that both forms of attention enhance perceptual processing similarly and are controlled by the same neural mechanisms (Gazzaniga et al., 1998). However, behavioral evidence indicates that voluntary and involuntary attention might have different time courses and consequences (Müller and Rabbitt, 1989; Berger et al., 2005).

In the present study, a cueing task was combined with electrophysiological measures, to compare voluntary and involuntary attention in identical stimulus conditions.

Previous work coupling electrophysiology with cueing paradigms mostly focused on event-related potentials (ERPs). The most consistent finding is an increase in early sensory potentials (P1 component) elicited by a cued target compared with an uncued target (Mangun and Hillyard, 1991; Hopfinger and Ries,

Received July 7, 2007; revised Sept. 24, 2007; accepted Sept. 25, 2007.

This work was supported by National Institutes of Health Grant MH 64458 (L.C.R., S.B.). We thank Brian Roach for the informative methodological exchange, and Michael Oliver and Drs. Robert T. Knight and Alexandra List for stimulating discussions. We would also like to thank Cheryl L. Shirley for her assistance in data collection.

Correspondence should be addressed to Ayelet Landau, University of California Berkeley, 3210 Tolman Hall, Berkeley, CA 94720-1650. E-mail: ayelet@berkeley.edu.

DOI:10.1523/JNEUROSCI.3092-07.2007

Copyright $\odot 2007$ Society for Neuroscience ～0270-6474/07/2711986-05\$15.00/0
2005). Later components may also be modulated, but the results are less consistent. Whereas some authors reported greater $\mathrm{N} 1$ on valid trials (Luck et al., 1994), others found the reverse (greater $\mathrm{N} 1$ on invalid trials) (Hopfinger and Ries, 2005). Hopfinger and West (2006) measured interactions between voluntary and involuntary attention manipulating the two types of attention concurrently. They find that although mutually affecting each other, voluntary and involuntary attention act on different stages of processing. However, in the procedure they used, the voluntary and involuntary cues were visually different, and were presented at different times in a given trial. Voluntary and involuntary attention effects have seldom been directly compared under equal stimulus conditions within the same ERP study. Studies that attempted this comparison revealed no marked differences in the amplitudes of either P1 or N1 (Doallo et al., 2005).

Previous work in both animals and humans examined the spectral content of the EEG signal (Gruber et al., 1999; Fries et al., 2001; Vidal et al., 2006; Fan et al., 2007) suggesting that activity in the gamma range $(>30 \mathrm{~Hz})$ (Tallon-Baudry and Bertrand, 1999) is modulated by attention. We therefore focused on the gammaband response as it relates to voluntary and involuntary attention. Whereas ERPs did not distinguish voluntary from involuntary attention, we report clear differences between these two attention systems in the gamma-band.

\section{Materials and Methods}

Task and procedures. Participants performed an easy face-discrimination task under conditions of voluntary and involuntary attention conditions. As shown in Figure 1a, one of two faces was presented immediately after a cue offset, either to the left or to the right of fixation, and the participants reported which face had been presented by pressing one of two keys. A peripheral cue preceded the target face in both voluntary and involuntary conditions. Voluntary attention was measured in the predictive-cue condition. In this condition, the face appeared more often 
in the cued location (70\% valid) than the uncued location. Involuntary attention was measured in the nonpredictive-cue condition. In this condition the cue location was unrelated to target location. Both conditions included target-absent trials, which allowed evaluation of cue-related activity in isolation of a target face. A third key was used to report the absence of a target. The only difference between the attention conditions was the proportion of valid, invalid, and cue-only trials. This design enabled examination of the physiological time course of cue and target processing for voluntary and involuntary attention without confounding physical stimulus parameters. The predictive-cue condition consisted of six 100-trial blocks separated by breaks. The nonpredictive-cue condition consisted of five 112-trial blocks (see Fig. 1a). Each participant completed one cue condition before starting the other cue condition, and cue conditions were counterbalanced between subjects. Each cue condition began with a short practice block (20 trials).

Note that in the predictive-cue condition the participant was encouraged to use the cue when it appeared, and anticipate the probable location of the target. In the nonpredictive-cue condition, participants were instructed to ignore the cues as they are independent of target location. Here, predictive- and nonpredictive-cue conditions were used as operational variables for voluntary and involuntary attention. With a predictive cue, voluntary attention would be allocated when the cue appears. With a nonpredictive cue, voluntary attention will not be allocated until the target appears.

Stimuli. Stimuli were presented on a 20 inch monitor. Seen from 155 $\mathrm{cm}$, the visual angle of each cueing square was $2.2^{\circ}$, and they were centered $2.7^{\circ}$ from a $0.4^{\circ}$ fixation cross. Faces appeared centered in one of the squares and were $2.4^{\circ}$ wide.

Participants. Sixteen undergraduates participated in the experiment for class credit. All participants had normal or corrected-to-normal visual acuity. Participants gave informed consent as approved by the University of California Berkeley Institutional Review Board.

EEG acquisition and processing. EEG was recorded using a Biosemi active-two system at a sampling rate of $256 \mathrm{~Hz}$ from 64 sites of a modified 10-20 system montage. Horizontal electro-oculographic (EOG) signals were recorded at the left and right external canthi, and vertical EOGs were recorded below the right eye. All electrodes were referenced off line to the tip of the nose. Preprocessing of the data was done in Brain Vision Analyzer. Trials with eye movement or blinks were removed from the data using an amplitude criterion of $\pm 150 \mu \mathrm{v}$ or lower. Ongoing EEG was segmented into epochs from $200 \mathrm{~ms}$ before cue onset to $1000 \mathrm{~ms}$ after cue onset of correctly performed trials. These data were then exported to EEGLAB (Matlab Toolbox) (Delorme and Makeig, 2004) for spectral analysis.

EEG spectral analysis. To measure the power at each frequency band and time point, the data were processed using the "timef" function of EEGLAB (Delorme and Makeig, 2004). For each experimental condition, $\sim 60$ randomly selected EEG epochs were convolved with Gaussianwindowed sinusoidal wavelets of two-cycle duration. At each frequency band, the mean spectral energy of the prestimulus baseline (from -200 to $-50 \mathrm{~ms}$, excluding the last $50 \mathrm{~ms}$ of fixation in which fixation changed color) was subtracted from the prestimulus and poststimulus timefrequency energy. The absolute power measure was converted to decibels $\left[10 \times \log \left(\mu \mathrm{V}^{2}\right)\right]$. Baseline levels in the two attention conditions were equivalent as revealed by a planned paired $t$ test $\left(t_{(15)}=1.05 ; p=0.92\right)$. The resulting time-frequency maps were averaged across trials for each subject to form the event-related spectral perturbation (ERSP) (Makeig, 1993). The individual subject maps were averaged to create grand average ERSP maps (see Fig. 2).

For statistical analysis, an unbiased time by frequency range of maximal gamma-band response was selected from the averaged data for cuerelated responses and target-related responses separately (YuvalGreenberg et al., 2007). The average power within these regions for each condition was then used as the dependent variable in an ANOVA with repeated factors. Because of the wide scalp distribution, the data were collapsed for analysis into three groups (anterior, central, and posterior) in each hemisphere (Gruber et al., 1999) (supplemental Fig. 1, available at www.jneurosci.org as supplemental material).

ERP analysis. Segmented data were averaged separately for each con- a.
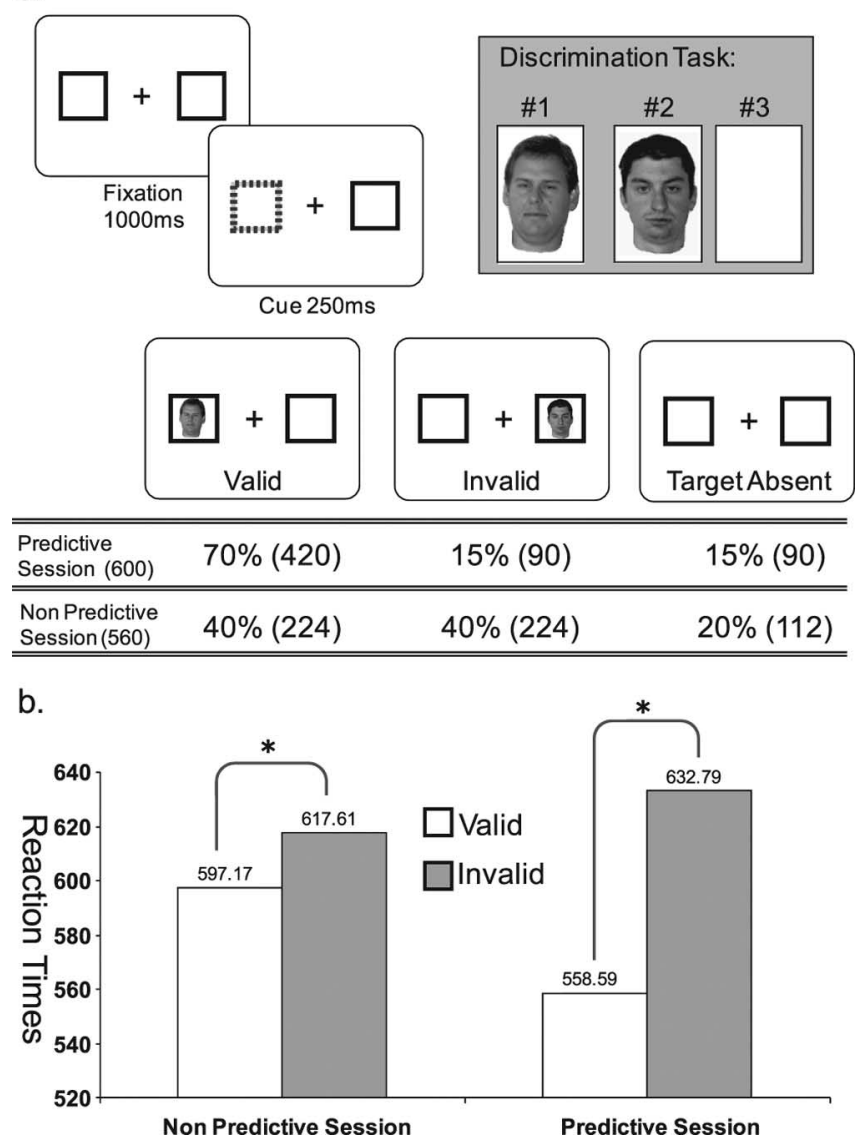

Figure 1. a, After $1 \mathrm{~s}$ of fixation, a cue (one rectangle changed to red, denoted by the dotted rectangle) was displayed for $250 \mathrm{~ms}$, followed by one of two face targets displayed for $300 \mathrm{~ms}$ or a blank screen. Bellow the illustration are the probabilities (and trial numbers) of each trial type (valid, invalid, cue-only) in the different cue conditions (predictive and nonpredictive). Participants indicated by a key press which face appeared or whether no face appeared. $\boldsymbol{b}$, Reaction time performance in milliseconds.

dition. Averaged waveforms were bandpass filtered $[0.8-17 \mathrm{~Hz} 24 \mathrm{db} / \mathrm{oct}$ (Zion-Golumbic and Bentin, 2006)] and baseline corrected from $100 \mathrm{~ms}$ precue onset. For each participant, the P1 peak was determined as local maximum between 80 and 150 ms post target onset and the peak of the N170 face-selective component (Bentin et al., 1996) was determined as local minimum between 130 and 220 ms. Amplitudes of these components at sites P8, PO8, and P10 over the right hemisphere, and the homolog sites over the left were included in the analysis. ANOVA with repeated measures with factors cue condition (predictive, nonpredictive), hemisphere (left, right), site (P7/8, PO7/8, P9/10), target side (left, right), and validity (valid, invalid) were manipulated as within subject variables and were performed on P1 and N170 amplitudes separately.

\section{Results}

\section{Performance}

Overall, discrimination of targets was faster for valid than invalid trials (Fig. 1b). The validity effect was greater in the predictive than nonpredictive-cue condition. These results were supported by a two-way ANOVA conducted for target-present trials. Overall, responses were slightly faster in the predictive than the nonpredictive-cue condition. However this difference was not significant $\left(F_{(1,15)}=3.32 ; p=0.09\right)$. The validity effect was significant $\left(F_{(1,15)}=30.29 ; p<0.001\right)$, and interacted with cue condition $\left(F_{(1,15)}=15.91 ; p<0.01\right)$. This interaction indicated that validity effects were larger in the predictive-cue condition than in the nonpredictive-cue condition. Planned paired com- 


\section{a. Cue Related Response}
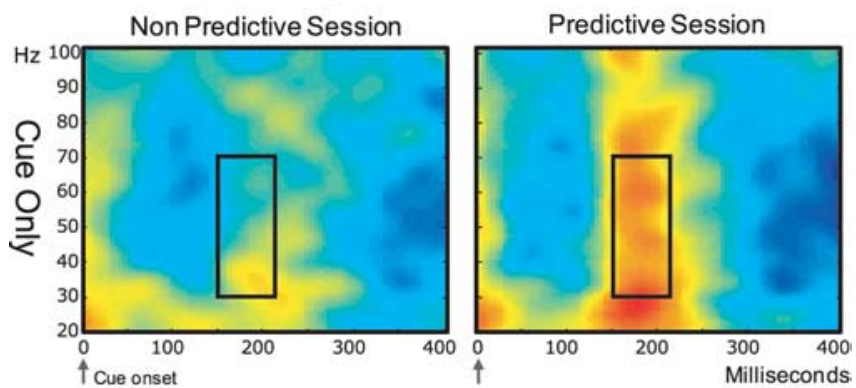

\section{b. Target Related Response}
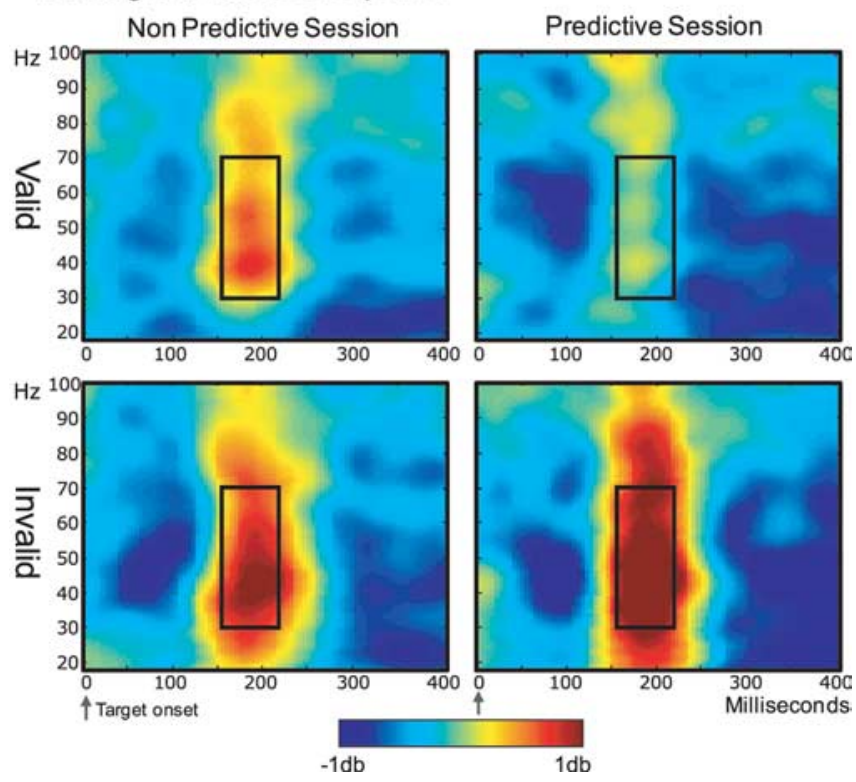

Figure 2. Time (x-axis)/frequency ( $y$-axis) plots for nonpredictive (left column) and predictive (right column) conditions. $\boldsymbol{a}$, Data from cue-only trials. Cue onsets at zero are marked with an arrow. $\boldsymbol{b}$, Data from target-present trials (valid and invalid in the first and second rows respectively). Target onsets at zero are marked with an arrow. Dark rectangles mark the time/ frequency window used in the statistical analysis.

parisons indicated that in both cue conditions the validity effect was significant $\left(t_{(15)}=5.14, p<0.001\right.$ for predictive and $t_{(15)}=$ $3.4, p<0.01$ for nonpredictive). For target absent trials, performance was identical for the two cue conditions ( $636 \mathrm{~ms}$ in both).

The percentage correct for valid and invalid trials after predictive cues were $94.7 \%$ and $92.4 \%$, respectively. The corresponding accuracy rates after nonpredictive cues were $95.2 \%$ and $93 \%$. ANOVA showed a main effect of validity in error rates (difference, $\left.2.25 \% ; F_{(1,15)}=17.30 ; p<0.01\right)$ and no interaction with cue condition $(F<1)$.

\section{Spectral analysis}

Activity in the gamma range (defined here as $30-70 \mathrm{~Hz}$ ) was averaged for the temporal window from 150 to $225 \mathrm{~ms}$ postcue onset to measure cue-related activity and from 150 to $225 \mathrm{~ms}$ post-target onset to measure target-related activity (Fig. 2) (timefrequency window selection process is as described in Materials and Methods section).

\section{Cue-related activity}

Analysis of target-absent trials showed that gamma-band power to cues was higher in the predictive than in the nonpredictive-cue condition (Fig. 2a). This observation was supported by an ANOVA with cue condition (predictive, nonpredictive), cue-side (left, right), hemisphere (left, right), and site (posterior, central, anterior). The main effect of cue condition was significant $\left(F_{(1,15)}\right.$ $=4.63 ; p<0.05)$ although the effects of all other factors were not (for the complete statistical analysis, see supplemental Table 1, available at www.jneurosci.org as supplemental material).

\section{Target-related activity}

The gamma activity elicited in the predictive-cue condition by invalid targets was higher than that elicited by valid targets. In the nonpredictive-cue condition, the gamma elicited by both target types (valid and invalid) was similar (Fig. 2b). An ANOVA with cue condition (predictive, nonpredictive), validity (valid, invalid), target side (left, right), hemisphere (left, right), and site (posterior, central, anterior) as within-subject factors showed a main effect of validity $\left(F_{(1,15)}=5.12 ; p<0.05\right)$, which interacted with cue condition $\left(F_{(1,15)}=4.70 ; p<0.05\right)$. Paired planned comparisons showed that in the predictive-cue condition gamma-band response was significantly greater on invalid then valid trials $\left(F_{(1,15)}=5.20 ; p<0.05\right)$. In the nonpredictive-cue condition this was not the case $\left(F_{(1,15)}=3.51 ; p=0.08\right)$. The trend in the nonpredictive case could be caused by a number of factors. ${ }^{a}$ Importantly, the significant interaction reflects the reliable differences in gamma activity between valid and invalid conditions for predictive and nonpredictive conditions.

In addition, the analysis revealed evidence for laterality in the gamma-band response in the predictive-cue condition. Both the hemisphere by target side interaction and the hemisphere by target side by site interaction were significant $\left(F_{(1,15)}=6.20, p<\right.$ 0.05 and $F_{(2,30)}=7.04, p<0.01$, respectively). Gamma power was higher over the hemisphere contralateral to the target location than over the ipsilateral hemisphere. The difference between the contralateral and ipsilateral response was significant for posterior and anterior sites $\left(F_{(1,15)}=7.16, p<0.05\right.$ and $F_{(1,15)}=$ $0.33, p<0.01$, respectively). Figure $3 a-c$ presents scalp distributions of the gamma-band response at three time points after target onset demonstrating the differences in the propagation of gamma over different scalp sites (supplemental Fig. 2, available at www.jneurosci.org as supplemental material). For the central sites the statistical test did not reveal significant laterality effects (target side by hemisphere effect $F_{(1,15)}=2.09 ; p=0.169$, not significant).

\section{ERPs}

P1 and N170 components did not differ between predictive- and nonpredictive-cue conditions ( $F<1$ for all relevant comparisons for both P1 and N170 components). Both components showed maximal response for sites located contralateral to the target side (hemisphere by target side significant interaction: $F_{(1,15)}=8.16$, $p<0.05$ for $\mathrm{P} 1$ and $F_{(1,15)}=8.67, p<0.05$ for N170). Consistent with previous reports in the literature (Mangun and Hillyard, 1991; Hopfinger and Ries, 2005), for both attention conditions, the P1 component was larger for valid conditions compared with invalid conditions when the target was presented in the contralateral visual field (supplemental material, available at www. jneurosci.org). This effect was supported by a significant hemisphere by target side by validity interaction $\left(F_{(1,15)}=17.14 ; p<\right.$ 0.01 ). For the N170, no such validity effect was found (hemi- 
a.

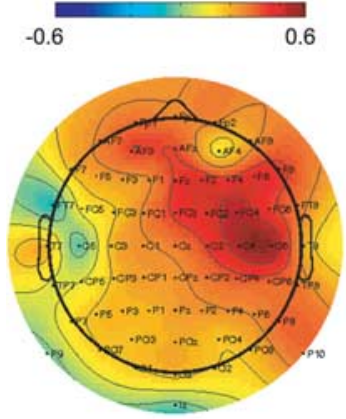

$141 \mathrm{~ms}$ b.

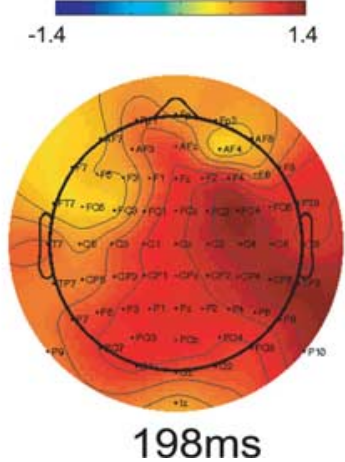

C.
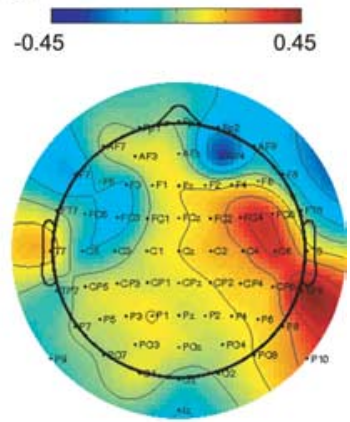

$236 \mathrm{~ms}$

Figure 3. $\boldsymbol{a}-\boldsymbol{c}$, The scalp distributions of gamma-band activity at 141, 198, and $236 \mathrm{~ms}$ on invalid trials in the predictive-cue condition. Frequencies $30-70 \mathrm{~Hz}$ were collapsed, and scales changed to emphasize differences in activity between different sites.

sphere by target side by validity: $F_{(1,15)}=1.23 ; p=0.28$, not significant).

\section{Discussion}

The present findings demonstrate that voluntary and involuntary attention have different effects on gamma-band response whereas both affect reaction times (RTs) similarly. These effects were manifested in two stages. The first was in response to the cue: only voluntary attention induced gamma-band increases to the cue alone (predictive-cue condition). The second was in response to targets: gamma-band increased whenever a voluntary shift of attention was required to a target. These patterns of gamma-response and the way they relate to voluntary and involuntary attention are detailed bellow.

Replicating many past studies, valid targets were detected faster than invalid targets regardless of the cue's predictive value. This is evidence that abrupt onsets of peripheral stimuli captured the observer's attention reflexively. When the cue was predictive, however, participants also oriented voluntarily to the cued location, which lead to enhanced validity effects. Because the addition of voluntary attention was the only difference between the two conditions, the higher gamma power elicited by the predictive relative to nonpredictive cues reflects EEG activity associated with voluntary attention. ${ }^{b}$

Responses to the targets differed depending on whether the cue was predictive or nonpredictive. In the nonpredictive condition, when the target appears, a voluntary attention shift to the target is required to carry out the discrimination task (for both valid and invalid trials). EEG response in those trials reveals that after a target appears in the nonpredictive condition, there is an increase in the gamma-band response (Fig. 2b). Hence for the nonpredictive-cue condition, response to the targets is supporting the correspondence between gamma-band response and voluntary shifts of attention.

In the predictive-cue condition, on valid trials, the cue summoned voluntary attention and there is no additional shift required to the target. In these trials, there was less gamma-band response because participants had already shifted their attention to the cued location. In contrast, in invalid trials, although a shift of attention has occurred to the predictive cue, attention has shifted to the wrong location and an additional shift of attention is required when the target appears. Examining the EEG response

${ }^{b}$ The time course of the voluntary attention EEG modulation found here is consistent with previous work measuring steady-state visual evoked potentials in response to an attentional cue in a sustained attention paradigm (Müller et al., 1998). in these trials reveals a marked increase in gamma-band response on invalid trials. Once again, EEG response in the gammaband seems to mirror the voluntary shift of spatial attention.

Previous investigations in the spectral domain of the EEG signals have suggested diverse roles for gamma activity which include perception, higher cognitive functions such as memory and object representation (Herrmann et al., 2004; ZionGolumbic and Bentin, 2006). TallonBaudry et al. (1999) have extensively examined the role of gamma-band activity in perceptual binding. In initial studies gamma activity was measured to stimuli requiring perceptual integration to form a visible object. However, because the object requiring binding was also the object of attention, it was unclear whether the factor eliciting the increases in induced gamma in these studies was perceptual binding, attentional selection or some combination of the two (Tallon-Baudry et al., 2005).

Previous work has shown that gamma-band response can be related to selective attention. Gruber et al. (1999) have reported increases in gamma-band activity at parieto-occipital sites contralateral to attended movement, and recently Vidal et al. (2006) have dissociated between the response to visual grouping and focused attention. In this study, however, participants were requested to selectively attend to a subset of the stimuli in a display for later report of the items orientation, a task involving a memory component in addition to the selective attention components. Fan et al. (2007) have previously reported increases in gamma-band response to a peripheral predictive cue, as we found here. The authors term this effect a spatial-orienting effect. However, in their study it is unclear whether these increases are caused by the peripheral sensory stimulation, the exogenous capture of attention by the peripheral cue or the voluntary deployment of attention to the cued location (cues were $100 \%$ predictive).

Our data rule out a sensory interpretation for these findings and suggest that increases in gamma-band response are related to voluntary rather than involuntary deployment of attention. Because mechanisms of selection and perceptual organization are thought to be highly interactive, perceptual binding and attention are difficult to tease apart. In the present study we circumvented this problem by observing the effects of voluntary and involuntary attention on gamma-band activity in the absence of sensory differences between these two attention conditions. It might be the case that the neural mechanisms that support voluntary shifts of attention are also involved in perceptual binding (Treisman and Gelade, 1980), however these questions await further investigation.

In contrast to gamma, ERPs did not discriminate between voluntary and involuntary attention. Similar to previous studies (Mangun and Hillyard, 1991; Hopfinger and Ries, 2005), we found evidence for early sensory processing for validly cued compared with invalidly cued locations (indicated by a larger P1 component on valid trials). However, this difference was largely unaffected by cue predictability (Doallo et al., 2005). This dissociation between higher frequency responses in the gammaband range and the low-frequency responses in ERPs suggest that high- and low-frequency EEG activity reflect different neural mechanisms.

Our findings in the gamma-band response relate to previous 
findings from single-unit recordings. In monkey V4, gamma activity correlates with attentional selection (Fries et al., 2001). Recordings in this study were limited to the extrastriate regions. Our data are complementary to the animal work, as they reveal contralateral responses to predictive cues and attended targets that initially appear in anterior regions and then propagate to posterior regions (Fig. 3, supplemental movie 1, available at www.jneurosci.org as supplemental material). Such contralateral frontoparietal distribution is consistent with evidence from both functional magnetic resonance imaging (fMRI) studies in humans and investigations in animals showing the relevance of cortical regions such as the frontal eye fields in selective voluntary attention (Corbetta, 1998; Buschman and Miller, 2007).

EEG Activity in the $\alpha$-band has been shown to be modulated by sustained voluntary attention (Worden et al., 2000, Thut et al., 2006). These modulations typically take $400 \mathrm{~ms}$ to develop after the onset of a spatial cue. It is possible that $\alpha$-band and gammaband responses interact, and future studies could address this issue using longer cue target intervals than those used here. The present study was designed to allow a direct comparison between voluntary and involuntary attention requiring relatively short cue-to-target intervals.

The differences in neural response between voluntary and involuntary attention conditions fit well with the hypothesis that they involve different mechanisms and suggest how these two types of attention may affect performance and perceptual processing. Previous support for this hypothesis can be found in both behavioral and imaging work. RT studies showed that involuntary attention effects dissipate rapidly and reverse at long cuetarget stimulus onset asynchrony, whereas the effects of voluntary attention on performance are sustained (Berger et al., 2005). Prinzmetal et al. (2005) suggest that there are several cases where voluntary attention affects accuracy whereas involuntary attention does not within identical stimulus conditions. fMRI studies also report differences between voluntary and involuntary attention, mostly in dorsal regions (Kincade et al., 2005). The blood oxygen level-dependent response to faces in the fusiform face area (Kanwisher et al., 1997) increased when a target face was presented at a cued location compared with an uncued location, but only if the cues were predictive of target location (M. Esterman and W. Prinzmetal, unpublished observation). The current EEG study provides insight to the temporal dynamics of voluntary and involuntary attention and reveals that gamma-band response reflects voluntary shifts of attention.

\section{References}

Bentin S, Allison T, Puce A, Perez E, McCarthy G (1996) Electrophysiological studies of face perception in humans. J Cogn Neurosci 8:551-556.

Berger A, Henik A, Rafal R (2005) Competition between endogenous and exogenous orienting of visual attention. J Exp Psychol 134:207-221.

Buschman TJ, Miller EK (2007) Top-down versus bottom-up control of attention in the prefrontal and posterior parietal cortices. Science 315:1860-1862.

Corbetta M (1998) Frontoparietal cortical networks for directing attention and the eye to visual locations: identical, independent, or overlapping neural systems? Proc Natl Acad Sci USA 95:831-838.

Delorme A, Makeig S (2004) EEGLAB: an open source toolbox for analysis of single-trial EEG dynamics including independent component analysis. J Neurosci Methods 134:9.

Doallo S, Lorenzo-Lopez L, Vizoso C, Holguín SR, Amenedo E, Bará S, Cadaveira F (2005) Modulations of the visual N1 component of eventrelated potentials by central and peripheral cueing. Clin Neurophysiol 116:807.

Fan J, Byrne J, Worden MS, Guise KG, McCandliss BD, Fossella J, Posner MI (2007) The relation of brain oscillations to attentional networks. J Neurosci 27:6197-6206.
Fries P, Reynolds JH, Rorie AE, Desimone R (2001) Modulation of oscillatory neuronal synchronization by selective visual attention. Science 291:1560.

Gazzaniga MS, Ivry RB, Mangun GR (1998) Cognitive neuroscience: the biology of the mind. New York: W. W. Norton.

Gruber T, Müller M, Keil A, Elbert T (1999) Selective visual-spatial attention alters induced gamma-band responses in the human EEG. Clin Neurophysiol 110:2074.

Herrmann CS, Munk MH, Engel AK (2004) Cognitive functions of gamma-band activity: memory match and utilization. Trends Cogn Sci 8:347.

Hopfinger JB, Ries AJ (2005) Automatic versus contingent mechanisms of sensory-driven neural biasing and reflexive attention. J Cogn Neurosci 17:1341-1352.

Hopfinger JB, West VM (2006) Interactions between endogenous and exogenous attention on cortical visual processing. NeuroImage 31:774-789.

Jonides J (1981) Voluntary versus automatic control over the mind's eye's movement. In: Attention and performance, Vol 9 (Long JB, Baddeley AD, eds), pp 187-204. Hillsdale, NJ: Erlbaum.

Kanwisher N, McDermott J, Chun MM (1997) The fusiform face area: a module in human extrastriate cortex specialized for face perception. J Neurosci 17:4302-4311.

Kincade JM, Abrams RA, Astafiev SV, Shulman GL, Corbetta M (2005) An event-related functional magnetic resonance imaging study of voluntary and stimulus-driven orienting of attention. J Neurosci 25:590-600.

Luck SJ, Hillyard SA, Mouloua M, Woldorff MG, Clark VP, Hawkins HL (1994) Effects of spatial cueing on luminance detectability: psychophysical and electrophysiological evidence for early selection. J Exp Psychol Hum Percept Perform 20:887-904.

Makeig S (1993) Auditory event-related dynamics of the EEG spectrum and effects of exposure to tones. Electroencephalogr Clin Neurophysiol 86:283.

Mangun GR, Hillyard SA (1991) Modulations of sensory-evoked brain potentials indicate changes in perceptual processing during visual-spatial priming. J Exp Psychol Hum Percept Perform 17:1057-1074.

Müller HJ, Rabbitt PM (1989) Reflexive and voluntary orienting of visual attention: time course of activation and resistance to interruption. J Exp Psychol Hum Percept Perform 15:315-330.

Müller MM, Teder-Sälejärvi W, Hillyard SA (1998) The time course of cortical facilitation during cued shifts of spatial attention. Nat Neurosci 1:631-634.

Posner MI (1978) Chronometric explorations of mind. Hillsdale, NJ: Erllbaum.

Prinzmetal W, McCool C, Park S (2005) Attention: reaction time and accuracy reveal different mechanisms. J Exp Psychol 134:73-92.

Tallon-Baudry C, Bertrand O (1999) Oscillatory gamma activity in humans and its role in object representation. Trends Cogn Sci 3:151.

Tallon-Baudry C, Bertrand O, Hénaff MA, Isnard J, Fischer C (2005) Attention modulates gamma-band oscillations differently in the human lateral occipital cortex and fusiform gyrus. Cereb Cortex 15:654.

Thut G, Nietzel A, Brandt SA, Pascual-Leone A (2006) $\alpha$-Band electroencephalographic activity over occipital cortex idexes visuospatial attention bias and predicts visual target detection. J Neurosci 26:9494-9502.

Treisman AM, Gelade G (1980) A feature-integration theory of attention. Cogn Psychol 12:97-136.

Vidal JR, Chaumon M, O’Regan JK, Tallon-Baudry C (2006) Visual grouping and the focusing of attention induce gamma-band oscillations at different frequencies in human magnetoencephalogram signals. J Cogn Neurosci 18:1850-1862.

Yuval-Greenberg S, Deouell LY (2007) What you see is not (always) what you hear: induced gamma-band responses reflect cross-modal interactions in familiar object recognition. J Neurosci 27:1090-1096.

Worden MS, Foxe JJ, Wang N, Simpson GV (2000) Anticipatory biasing of visuospatial attention indexed by retinotopically specific alphaband electroencephalography increases over occipital cortex. J Neurosci 20:RC63.

Zion-Golumbic E, Bentin S (2006) Dissociated neural mechanisms for face detection and configural encoding: evidence from N170 and induced gamma-band oscillation effects. Cereb Cortex 17:1741-1749. 